\title{
Questes
}

Revue pluridisciplinaire d'études médiévales

Journée d'étude 1 - Trier, classer, organiser | 2022

Trier, classer, organiser

\section{Ordonner son activité et son entreprise : la gestion des archives privées de Jean Teisseire, artisan du $\mathrm{XIV}^{\mathrm{e}}$ siècle}

\section{Mélanie Dubois Morestin}

\section{(2) OpenEdition}

1 Journals

Édition électronique

URL : https://journals.openedition.org/questes/6010

DOI : 10.4000/questes.6010

ISSN : 2109-9472

Éditeur

Les Amis de Questes

Édition imprimée

Pagination : 173-189

ISSN : 2102-7188

Référence électronique

Mélanie Dubois Morestin, «Ordonner son activité et son entreprise : la gestion des archives privées de Jean Teisseire, artisan du xiv siècle », Questes [En ligne], Journée d'étude 1 - Trier, classer, organiser | 2022, mis en ligne le 31 janvier 2022, consulté le 21 février 2022. URL : http://

journals.openedition.org/questes/6010 ; DOI : https://doi.org/10.4000/questes.6010 


\title{
Ordonner son activité et son entreprise : la gestion des archives privées de Jean Teisseire, artisan du xiv ${ }^{\mathrm{e}}$ siècle
}

\author{
Mélanie DUBOIS MoRESTIN \\ LAMOP, Université Paris 1-Panthéon-Sorbonne
}

Écrire chaque jour, trier, classer et organiser les écrits ainsi produits, telles sont les activités quotidiennes de Jean Teisseire, artisan cordier avignonnais de la fin du xiv ${ }^{\mathrm{e}}$ siècle.

L'ensemble de sa carrière, depuis 1347, date à laquelle il prend son indépendance et se met à son compte en tant qu'artisan cordier, jusqu'à 1384, date de sa mort sans héritier - sinon la ville qui devient héritière universelle de ses biens -, nous est connu grâce à un dossier documentaire très complet. Le marchand tient en effet des écrits de gestion, actes de la pratique et pour certains du for privé, actualisés et classés en fonction de l'utilité et de l'utilisation des documents produits. Il tenait ainsi la raison de son ouvroir, entendu au sens de ratio, c'est-à-dire au sens de gestion, de comptabilité et de gestion pratique. Cette activité scripturale a produit un ensemble de manuels, livres de raison mêlant comptabilités, transactions de diverses natures et mentions personnelles ${ }^{1}$. Un seul nous est parvenu,

\footnotetext{
${ }^{1}$ Sur les livres de raison, sans prétendre à l'exhaustivité, quelques références peuvent être utiles : voir notamment Michel Cassan, Jean-Pierre Bardet et François-Joseph Ruggiu, Les écrits du for privé, objets matériels, objets édités, Limoges, Pulim, 2007, ou encore Par écrit, ethnologie des écritures quotidiennes, dir. Daniel Fabre, Martin La Soudière et Claudie Voisenat, Paris, Éditions de la maison des sciences, 1997. Du côté italien, on consultera avec profit I libri di famiglia in Italia, Vol. 1 : Filologia e storiografia letteraria, dir. Angelo Cicchetti et Raul Mordenti, Roma, Edizioni di storia e letteratura, 1995. Enfin, la synthèse produite par Sylvie Mouysset est un point d'entrée
} 
couvrant les années 1370 à 1377, sur un ensemble de près d'une vingtaine d'ouvrages produits par le cordier, et archivés dans sa maison - comme en atteste l'inventaire de ses biens après décès. En parallèle de ce livre, des actes notariés qu'il conservait et cotait, des comptabilités personnelles et institutionnelles, viennent donner un panorama complet de l'activité du cordier et surtout de ses techniques scripturales lui permettant de classer, lister et organiser les écrits produits ${ }^{2}$. Cette gestion se devait d'être efficace et pratique en raison de ses multiples activités en tant qu'artisan, marchand, membre influent de la ville d'Avignon ${ }^{3}$ et propriétaire de terres et de maisons.

Dans un premier temps, je présenterai le système des archives privées de Jean Teisseire, comme organisation interne de son activité et de son entreprise ${ }^{4}$ en insistant sur les temps d'écriture et les pratiques

commode pour une vue couvrant à la fois Moyen Âge et Modernité : Papiers de famille : Introduction à l'étude des livres de raison (France, $X V^{e}-X I X^{e}$ siècle), Rennes, PUR, 2008.

${ }^{2}$ Les archives de Jean Teisseire se trouvent rassemblées en deux principaux endroits : Archives départementales de Vaucluse, Dépôt Avignon, II 229 et II 330, mais également - pour les archives notariées - dans les Grandes Archives, Classement Pintat, boîte $\mathrm{n}^{\circ} 96$. On trouve également des ressources isolées, à la Bibliothèque Ceccano, manuscrit $n^{\circ} 5380$, pour un recueil de pièces, dont un règlement de comptes par Jean Teisseire du $1^{\text {er }}$ octobre 1362. Certaines comptabilités rédigées par Jean Teisseire peuvent également être consultées : Boîte 96, n 3270 ou encore Boîte 96 $\mathrm{n}^{\mathrm{o}} 3156$. Enfin, il faut signaler des mentions de la main de Jean Teisseire sous la forme de notes dorsales, pour des actes notariés conservés dans les boîtes 20, 82, 83 et 97 . Certains actes notariés, non conservés par Jean Teisseire lui-même, le mentionnent néanmoins, ainsi aux boîtes $3,7,8,20,21,22,25,34,46,82$ et 83 dans les pièces, respectivement, $\mathrm{n}^{\circ} 77,254,284,640,56,680,747,983,1508,2696$ et 2717. Des éléments supplémentaires peuvent être consultés dans les comptabilités de la ville d'Avignon aux cotes suivantes: AC Avignon, série CC, le CC1, CC21, CC61, CC1005, CC1010 et CC1107 ou encore dans la série D, le DD9 et DD10, livre censier de la ville comme héritière des biens de Jean Teisseire en 1425.

${ }^{3}$ Sur la société avignonnaise du XIV siècle, voir les travaux d'Anne-Marie Hayez, et

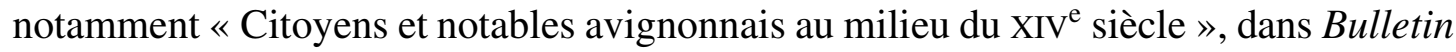
philologique et historique du comité des travaux historiques et scientifiques, 19821984, p. 199-219.

${ }^{4}$ Voir Mélanie Dubois-Morestin, Pratiques scripturales et pratiques économiques : les archives privées de Jean Teisseire, artisan avignonnais $d u$ XIV siècle, thèse d'histoire sous la direction de Laurent Feller, Université Paris I, 2015. Voir également 
concrètes de rédaction. Dans un second temps, je m'attacherai à montrer l'importance des listes et des ruptures du discours au sein du livre de raison, par la production de feuillets volants et d'écritures isolées qui permettent une gestion plus immédiate de certaines données. Ces éléments devraient permettre, par le croisement avec d'autres types d'archives privées d'artisans provençaux - notamment ceux de Paul de Sade, d'Olivier Jacme, ou encore d'Hugues de $\operatorname{Sade}^{5}$-, de pousser plus avant l'analyse des pratiques scripturales de ces groupes de notables urbains.

\section{Ordonner ses écrits}

Le livre de raison de Jean Teisseire contient des notices brèves résumant ses principales activités, rédigées de sa main, à la première personne. Ces notices sont longues d'une dizaine de lignes en général, et reprennent l'ensemble de ses transactions personnelles et professionnelles en tant que père, mari et artisan-marchand. Le livre de raison fonctionne comme un point central, quasi index, à partir duquel s'organise l'ensemble des archives privées du cordier, dans lequel il se repère par un système particulièrement ordonné ${ }^{6}$.

\footnotetext{
«Administrer et gérer par l'écrit: les pratiques scripturales de Jean Teisseire, petit entrepreneur du XIV siècle », dans Administrer par l'écrit (Actes de la Journées d'étude du 30-31 janvier 2015), Paris, Publications de la Sorbonne, Paris, 2019.

${ }^{5}$ Ces personnages ont fait l'objet d'études séparées, mais pas de synthèse, ce que s'attache à faire ma thèse (voir supra note 4). On peut cependant consulter les ouvrages suivants les concernant: Alphonse Blanc, Livre de comptes de Jacme Olivier, marchand narbonnais du XIV siècle, Paris, Picard, 1899 ; sur la famille des Sade, vori Henri Bresc, Le livre de comptes de Paul de Sade, 1390-1394, Paris, Éd. du Comité des travaux historiques et scientifiques, 2013. Sur Hugues de Sade, aucune étude n'a encore été menée.

${ }^{6}$ Sur la question de la gestion par l'écrit dans des entreprises du Moyen Âge, voir l'étude de Agnès Pallini-Martin, « La gestion et la maîtrise du temps et de l'espace dans la pratique marchande de la compagnie Salviati de Lyon autour de 1500 », dans Mélanges de l'École Française de Rome-Italie et Méditerranée modernes et contemporaines, 125, 2013, mis en ligne le 21 octobre 2013, http://mefrim.revues/org/1245. Voir également Laure-Hélène Gouffran, «L'Écriture comptable d'un marchand provençal au tournant du $\mathrm{XV}^{\mathrm{e}}$ siècle : les comptabilités de
} 
Il s'agit tout d'abord d'un système de références internes, permettant des retours et va-et-vient à l'intérieur de ses archives et de ses manuels. Ces renvois lui permettent de rappeler ou d'anticiper des transactions passées ou ultérieures de façon à les mettre à jour. Lorsqu'une affaire est soldée, Jean Teisseire l'indique et peut ainsi canceller la notice qui avait été inscrite, tout en renvoyant à la page à laquelle le paiement - dans la plupart des cas - a été fait. De cette manière, une double temporalité se met en place : en complément d'une composition globalement chronologique, entrecoupée - on le verra en seconde partie - de dossiers thématiques et personnels, c'est également une composition plus complexe et réfléchie qui est ainsi organisée. L'écriture prévoit en effet le temps de la relecture et des mises à jour par le biais d'un formulaire adapté : lors d'une référence à une transaction passée, il inscrit : «escrig atras en XXX cartas d'esto cartolari » (j'ai écrit précédemment à la page 30 de ce cartulaire) ; lors d'une référence à une transaction ultérieure qui vient compléter ou achever une affaire en cours : «mudem avant en cartas... » (j'ai réglé cela plus loin à la page...) / « aiso fon afinat ».

Ces mentions supposent des vérifications et va-et-vient dans l'ouvrage. Elles sont parfois mises en valeur par un encadré ou une croix de façon à rendre la lecture plus aisée et plus efficace - ce qui est l'objectif principal du cordier dans son écriture de gestion.

Si l'ouvrage est très minutieusement tenu, quelques erreurs s'y glissent parfois et nous permettent de mieux comprendre la méthode du cordier. En effet, il laisse parfois des blancs permettant d'apporter des ajouts ou précisions : même s'il peut certes s'agir d'oublis, on peut malgré tout penser qu'il pouvait ainsi revenir sur son écrit pour le compléter avec un montant, ou un numéro de page. Ces blancs nous prouvent que ces

Bertrand Rocafort d'Hyères », Comptabilités, 4, 2012, mis en ligne le 24 avril 2014, http://journals.openedition.org/comptabilites/1170. 
relectures sont envisagées à l'avance et supposent également l'existence d'écrits annexes dans lesquels certains éléments seraient inscrits, sortes de brouillons permettant de mémoriser l'essentiel avant la mise au propre dans le livre de raison. Une mémoire sélective est donc à l'œuvre, dans l'écriture tout comme dans la relecture : le système de l'écrit est chez Jean Teisseire un système toujours en construction, et le livre de raison n'est pas un ouvrage achevé. Il s'agit d'un outil pratique et pragmatique, qui est en évolution, constamment modifié, complété et annoté. Il est le résultat d'une pensée qui construit et œuvre, mais pas un point d'achèvement.

En analysant ces systèmes de va-et-vient et de relectures, on peut déterminer l'existence de plusieurs unités temporelles d'écriture et d'organisation des écrits. Le premier temps d'écriture est fait de notices sur papiers volants, qui sont parfois conservés, comme l'indiquent les documents de Jean Teisseire, mais que nous n'avons pas nécessairement retrouvés dans les archives. Le deuxième temps d'écriture consiste en l'inscription de la transaction, de manière quasi immédiate, dans le livre de raison. Le troisième temps d'écriture peut inclure une réflexion ou un retour sur les écritures, de manière à établir des comptes ou des listes, dont nous parlerons dans la seconde partie. Le quatrième temps d'écriture est celui des mises à jour, corrections, annotations, cancellations.

Le système est également fait de références externes, donnant une vue sur la manière dont s'organise l'ensemble du corpus du marchand, conçu comme un tout cohérent. Si la cohérence est celle des archives, elle est également celle de l'organisation de sa société et entreprise : ainsi, l'écriture et les documents offrent une cohérence spatiale et temporelle à l'ensemble des activités - très diverses - de l'entrepreneur. D'autre part, le testament du cordier ainsi que son inventaire après décès nous offrent une vue particulièrement précise du classement matériel des archives nombreuses, dans des coffres, caisses et diverses pièces de sa boutique et 
de sa maison ${ }^{7}$. Ce classement nécessite un système de cotations que le cordier met au point et qui, s'il reste obscur dans son utilisation exacte, n'en est pas moins innovant. Par l'ensemble de cette organisation, c'est ainsi tout un ensemble de documents tenus qui apparaissent (tableau 1) :

- des livres antérieurs ;

- des livres ultérieurs ;

- des livres annexes/spécialisés ;

- des notices écrites à part.

Ce sont les mêmes formules que les renvois internes qui apparaissent ainsi, mais renvoyant donc à d'autres «cartulaires » ou d'autres écrits, rangés dans ses archives, ou au contraire contenus dans le livre de raison lui-même comme c'est le cas pour certaines notices ${ }^{8}$. Ces formules, par l'analyse précise de leurs occurrences et du lexique employé, permettent de retracer le portrait complet des archives privées de Jean Teisseire et de leur organisation.

\section{Tableau 1}

Archives privées de Jean Teisseire, récapitulatif ${ }^{9}$

\footnotetext{
${ }^{7}$ Archives départementales de Vaucluse, Dépôt Avignon, Grandes Archives, boîte 96.

${ }^{8}$ Le terme « cartulaire » employé ici s'entend comme la traduction du terme latin utilisé par Jean Teisseire lui-même, à savoir cartularium. Il s'agit pour lui d'un recueil des cartas établies par les notaires, c'est-à-dire des grosses rédigées par ces derniers. Ce terme s'entend donc à la manière des registres des notaires.

${ }^{9}$ «Polisias » signifie ici «Quittances ». Le terme vient du grec polis, la cité. Ce sont au départ des écrits émanant de la ville, sous forme de quittances. Par extension, l'ensemble des notaires, marchands et hommes de l'écrit, manient ce type de notices.
} 


\begin{tabular}{|l|l|l|l|l|}
\hline Livres de raison & Livres spécialisés & Polisias & $\begin{array}{l}\text { Comptabilités } \\
\text { particulières }\end{array}$ & $\begin{array}{l}\text { Actes notariés } \\
\text { conservés }\end{array}$ \\
\hline 1356 & Livre des cordes & Diverses & Peyret Ortolan & Divers \\
\hline 1367 & Livre de l'ouvroir & & Douceline de Saze & \\
\hline 1369 & Livre vert & & $\begin{array}{l}\text { Succession de } \\
\text { Peire d'Uzès }\end{array}$ & \\
\hline $1370 / 1376$ & Petit cartulaire & & & \\
\hline 1378 & & & & \\
\hline
\end{tabular}

Cette organisation est également visible dans l'inventaire de ses biens après-décès. Il permet de repérer les caisses et coffrets qui contiennent ses archives, révélant au moins 20 cartulaires qui sont mis au jour : des cartulaires généraux, de la même taille et du même nombre de feuillets que celui que l'on a conservé pour les années 1370 à 1377. Cette donnée indique qu'il s'agissait du même style d'ouvrages, répondant aux mêmes objectifs : des livres généraux, servant de point d'entrée dans un système fait de livres annexes et d'actes notariés classés. À ces cartulaires généraux, réceptacles des affaires courantes, s'ajoutent des livres spécialisés : livre des cordes, livres de comptabilité, livre contenant la raison de l'ouvroir et autres livres dont il est difficile de connaître précisément le rôle mais qui entrânent une accumulation documentaire importante. Cette accumulation, utile et nécessaire au cordier pour ordonner, classer, de façon pratique et efficace, est gérée et maîtrisée grâce au livre de raison qui est un point d'entrée commode dans l'ensemble des archives. La masse documentaire est également administrée par des cotations chiffrées, qui apparaissent sur certains documents, ou encore par le biais du classement matériel référencé, tel qu'il apparaît dans l'inventaire après décès - mais qui reste difficile à saisir dans toutes ses subtilités. 
Ainsi, le livre de raison fonctionne comme un livre comptable et un livre de famille, en lien avec un ensemble de manuscrits auxquels il fait référence mais également comme un index permettant de comprendre les archives annexes tenues de manière complémentaire :

\section{Tableau 2}

Conservation des archives de Jean Teisseire

\begin{tabular}{|l|l|l|l|}
\hline Type de pièce & Type documentaire & Nombre & $\begin{array}{l}\text { Moyen } \\
\text { conservation }\end{array}$ \\
\hline Chambre & $\begin{array}{l}\text { Cartulaires et actes } \\
\text { notariés }\end{array}$ & $\begin{array}{l}8 \text { cartulaires } \\
1 \text { livre de parchemin } \\
\text { Des instruments et } \\
\text { papiers }\end{array}$ & Coffres \\
\hline Cour voisine & Cartulaires & $\begin{array}{l}6 \text { cartulaires } \\
2 \text { cartulaires }\end{array}$ & Coffres \\
\hline $\begin{array}{l}\text { Chambre voisine de la } \\
\text { cuisine }\end{array}$ & $\begin{array}{l}\text { Cartulaires et actes } \\
\text { notariés }\end{array}$ & $\begin{array}{l}5 \text { cartulaires } \\
\text { Actes nombreux }\end{array}$ & Aucune précision \\
\hline
\end{tabular}

\section{Ordonner pour organiser une société}

Les préoccupations gestionnaires, liées à l'économie domestique du cordier tout autant que professionnelle, expliquent l'organisation scripturale du dossier, mais elles doivent également être mises en relation avec une volonté d'organisation d'une entreprise, de boutiques, de voyages et d'employés nombreux. S'il ne s'agit pas d'un ordonnancement du monde, il s'agit pourtant bien d'une organisation de la société urbaine, constituée d'un groupe de notables urbains : c'est ainsi l'ordonnancement d'un monde. L'écriture et l'établissement de listes permettent en effet au cordier de réduire l'incertitude qui règne sur toute activité économique en 
organisant son personnel, les travaux à accomplir ou à fournir, les dépenses en cours ou à venir, et d'une manière générale en rendant possible la prévision de ses affaires sur un temps relativement long. Il rédige ainsi des notices et des listes résumant les activités de ses facteurs, qui travaillent pour son compte, mais également les prix des produits qu'il vend, ou encore les dettes qui restent à payer par ses débiteurs, rappelées en tête du manuscrit du livre de raison. Certains dossiers comptables présentent également des caractéristiques particulières, interrompant le cours du livre de raison - habituellement chronologique - afin de permettre un classement visuel plus simple et efficace de certaines transactions ou de certaines relations d'affaires. Ces interruptions dans le discours et la pratique de listes dans un but précis indiquent la manière dont le cordier utilise l'écrit pour organiser sa pensée et son administration. C'est bien la nature performative de l'écriture et de la disposition de cette dernière qui est mise en évidence ici, comme l'indiquait Clément Lenoble dans l'analyse du milieu franciscain, lorsqu'il envisage leur rôle dans l'économie de la ville, par leurs relations avec les marchands.

Je m'arrêterai donc ici sur un ensemble d'exemples de ces listes et feuillets, souvent insérés dans le livre de raison. Une liste de créances est présente en tête de manuscrit, rédigée par le cordier et mise en exergue par lui (fig. 1) : elle est au début du livre de raison, dans le manuscrit II330, avant même la partie foliotée et avant les comptabilités de l'hôpital SaintBénezet dont Jean Teisseire avait la charge. Il s'agit donc d'une partie spécifique, nécessitant une prise en compte particulière par l'historien. Par sa présentation, Jean Teisseire introduit une rupture graphique dans l'ensemble du livre de raison. La liste débute avec le titre suivant :

Dans le manuel de l'année 1369 qui a été commencé se trouvent les dettes qui suivent, que l'on peut garder en mémoire, provenant du 
cartulaire de l'année 1369, telles qu'elles sont écrites ci-dessous ${ }^{10}$.

La rupture graphique est marquée par ce titre, encadré et signalé par une bordure tracée sur la largeur de la page. La liste cherche à indiquer l'ensemble des débiteurs de Jean Teisseire : ainsi chaque entrée mentionne un nom, un montant, répertoriés sur une ou deux lignes, suivies d'espaces laissés blancs afin de faciliter la lecture. La liste est mise à jour par Jean Teisseire, lorsqu'il peut indiquer un paiement (avec la date ainsi que la page du livre dans laquelle cette dette est soldée). Si la rupture graphique est claire dans la présentation de la liste, elle est en revanche construite de manière très homogène et indique, après le nom et le montant, la page du manuscrit dans lequel trouver la référence à la transaction, ainsi que l'année à laquelle la créance a été contractée en marge. De cette façon, un véritable tableau est mis en place, parfaitement lisible et réglé : l'ordonnancement visuel ainsi créé va de pair avec une organisation gestionnaire, qui recherche l'efficacité pratique de mémorisation et de gestion. Cette rupture graphique n'a cependant pas pour but d'établir un compte qui permettrait de dresser un bilan chiffré. Il n'y a pas de somme des montants dus : le but est bien plus de classer, de retenir les informations nécessaires et de créer un index. C'est un moyen d'ordonner ses relations et surtout de retenir les mauvais payeurs : ceux qui n'ont jamais remboursé ne sont plus jamais apparus dans le livre de raison, comme si la liste était une forme de fichier d'identification.

Au-delà de la présentation graphique de la liste, les choix d'écriture de Jean Teisseire retiennent l'attention : la liste a été conçue en parallèle de l'écriture du cartulaire de l'année 1369. Jean Teisseire a ainsi copié les dettes que contenait le cartulaire 1367, pour éviter d'avoir à le consulter

\footnotetext{
10 «En lo manoal de l'an LXIX que fon comensat son los deutes cals sen sieguon cals hon pot recobrar dal cartolari de l'an MCCCLXIX son aquestos deutes que sen sieguon aisi desot »
} 
trop régulièrement alors même que ce cartulaire était probablement complet. Au moment de la rédaction du cartulaire de 1369, il continuait à inscrire les dettes restant à régler sur un document à part, cette liste. Ainsi, tout en notant ses transactions professionnelles et personnelles dans son livre, il opérait un reclassement documentaire dans la liste de dettes, de manière régulière et organisée. C'est ainsi que la tenue de cette liste prend le caractère d'un quasi-index, évitant les retours trop fréquents sur l'ouvrage complet, ce qui rendrait la manipulation plus complexe et laborieuse. La liste est un des outils qui s'intègrent à la gestion du livre principal. Sans que ce dernier ne soit un maître-livre, à la manière des compagnies marchandes, il est néanmoins le nœud de la gestion de l'ouvroir de l'artisan et de sa maisonnée.

Ainsi, la tenue parallèle des deux écrits, la liste d'une part et le cartulaire dans son ensemble de l'autre, permet de comprendre les quelques erreurs qui apparaissent dans les feuillets 7 et 8 , les derniers de la liste de dettes : des notices renvoient à des transactions de décembre 1369 et non pas spécifiquement à des créances. Il tenait donc bien les deux écrits dans le même temps, en parallèle. En finissant le cartulaire de 1369, il complétait encore cette liste de dettes, ce qui l'a amené à quelques confusions. 


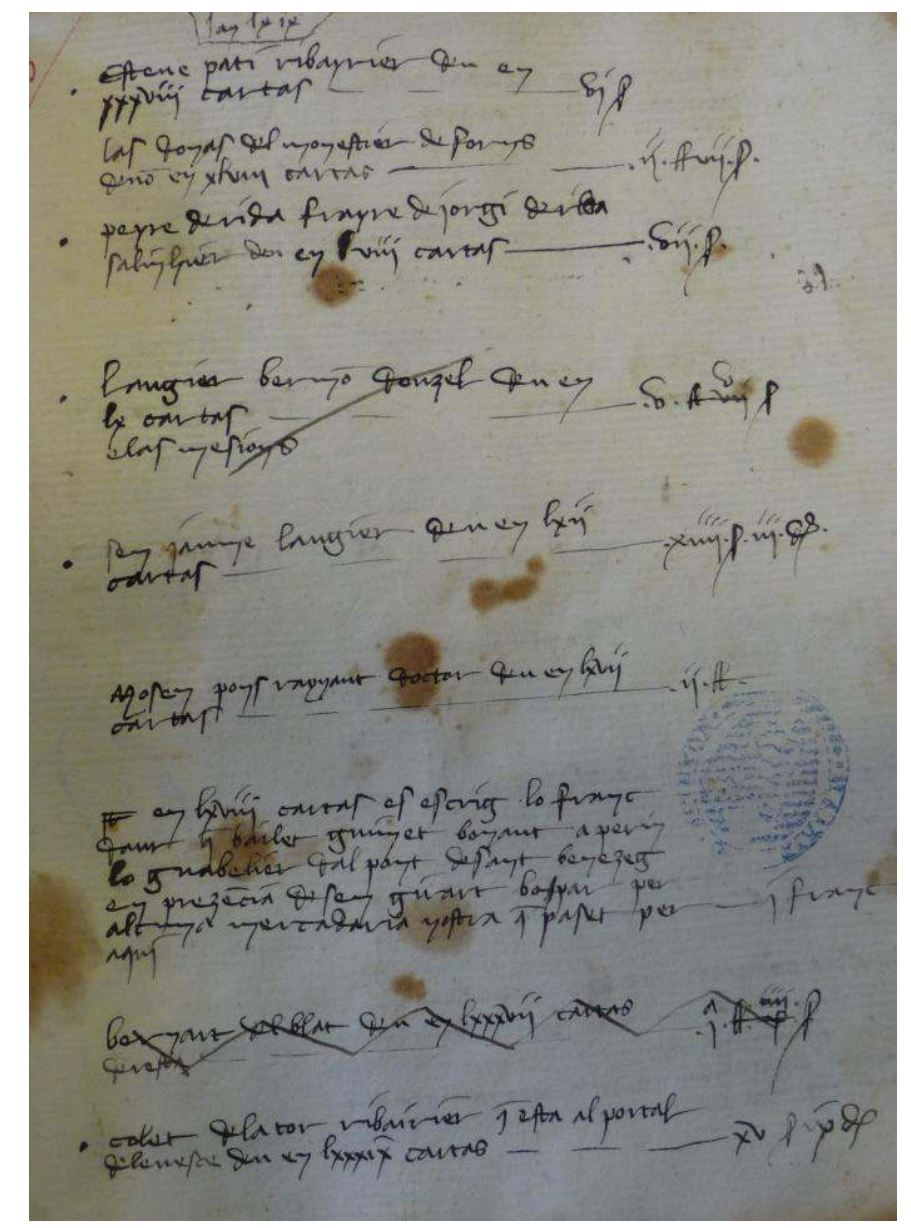

Fig. 1. Premier feuillet de la liste de créances, II330

Un autre exemple de rupture graphique dans l'ordonnancement des archives de Jean Teisseire est constitué par un dossier comptable consacré à un Olivier Amoros, un facteur de Jean Teisseire durant les années 1360 qui fut notamment envoyé à Barcelone pour le compte du cordier. Il semble qu'en 1369, après un dernier voyage à Barcelone pour le compte de son maître, il s'installe à son compte et possède son propre ouvroir. Un compte final est alors établi entre les deux personnages, aux folios $43 \mathrm{r}^{\circ}$ jusqu'à $45 \mathrm{v}^{\circ}$, au sujet de ce voyage en Espagne et d'autres missions effectuées pour le compte de Jean Teisseire. Ce compte se distingue nettement du reste du livre de raison: le cours chronologique habituel s'interrompt pour donner lieu à une liste, sur plusieurs folios donc, de toutes les entrées s'ajoutant au débit ou au crédit d'Olivier Amoros, avec 
le renvoi aux cartulaires dans lesquels ces transactions peuvent se trouver à l'origine. La rupture graphique est marquée par des retours à la ligne pour chacune des nouvelles entrées, mais surtout par l'utilisation - de manière unique dans un livre de raison - de marqueurs classificatoires, qui sont ici des lettres. Ainsi, les lettres $a, b, c, d$, sont inscrites en marge à gauche, au début de chacune des entrées. En marge, à droite, sont reportés les montants, très clairement, ce qui permet de faire les comptes de manière beaucoup plus efficace et pratique. Un titre est donné à la page, sous la mention «Olivier Amoros doit ce qui suit ici », afin que la lecture en soit facilitée. Enfin, un autre signe visuel organise la liste : en bas de page, on trouve une phrase qui est unique dans ce livre et indique qu' il faut « tourner la page ». L'interruption dans le texte est claire et correspond à un ordonnancement visuel qui cherche en même temps à ordonner les comptes, à mettre en ordre une relation entre deux hommes et à mettre en liste des événements dont la cohérence spatiale et temporelle était parfois délicate à saisir. Le montant total est ici indiqué puisqu'il s'agit d'un compte final et s'établit à 415 florins dus par Olivier Amoros pour affaires faites pour le compte de l'ouvroir de Jean Teisseire, pour un ensemble de marchandises diverses (cuivre, graisse, étoffes, vêtements, etc.). Le compte établi avec Olivier Amoros est le seul de ce type dans le livre de raison qui n'avait manifestement pas pour objectif de dresser des bilans chiffrés, sauf pour des transactions spécifiques. 


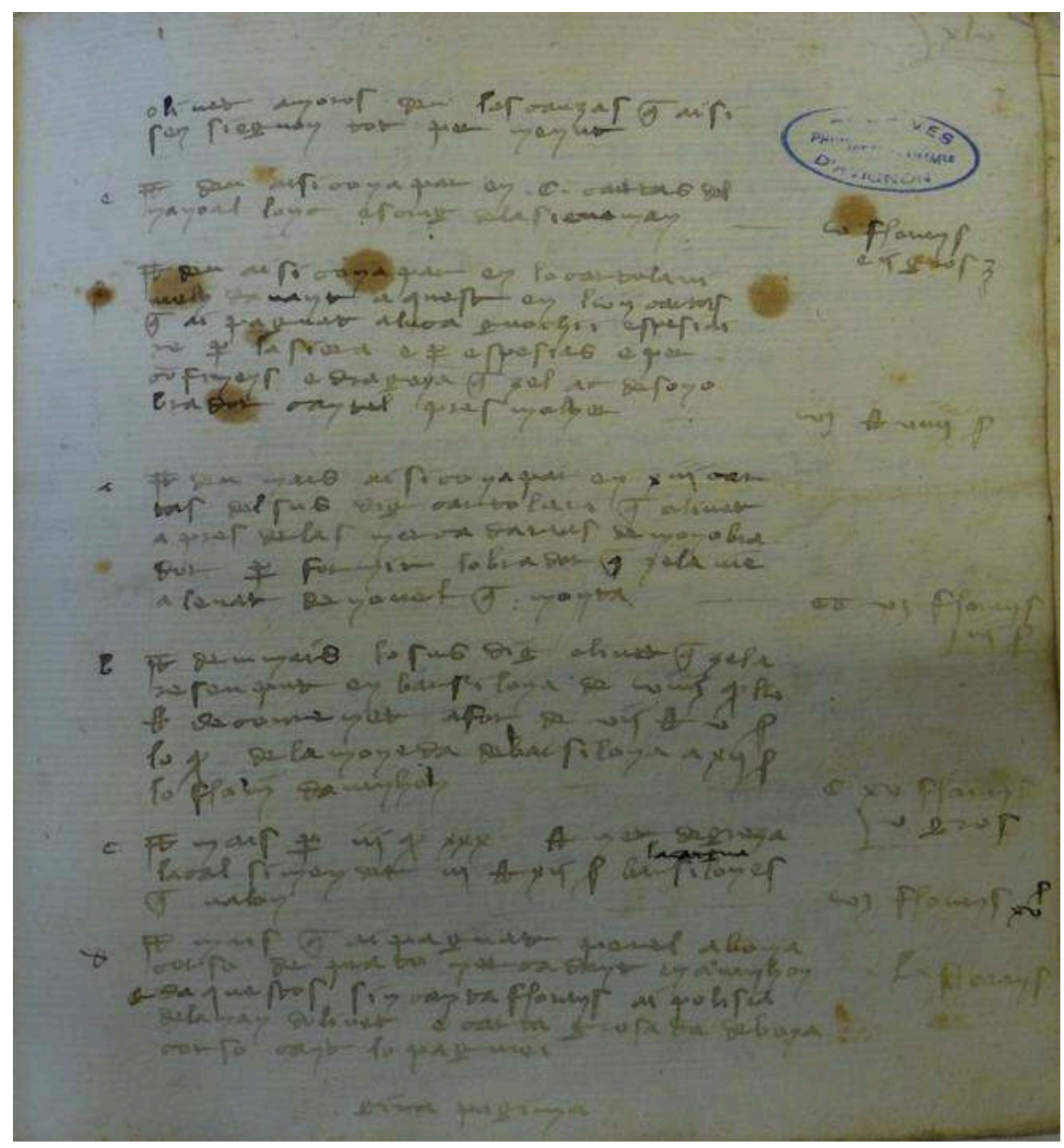

Fig. 2. Comptes d'Olivier Amoros

Ces comptabilités spécifiques sont en général inscrites sur des feuillets qui ne sont pas repris dans le livre de raison, mais qui nous sont parvenus par des hasards de la conservation, lorsque Jean Teisseire les a insérés dans le livre, et qu'ils y ont été conservés. Ces papiers sont des notices rédigées par Jean Teisseire ou des notaires, pour rappeler l'essentiel d'une transaction notariée dont le cordier conservait par ailleurs la grosse, ou bien des memoranda reprenant certaines transactions particulières, destinés à être transmis à une personne par exemple, ou bien destinés à faire mémoire. Ils ont probablement été utilisés lorsque le livre de raison n'était pas immédiatement disponible, pour garder une trace, avant que la transaction ne soit reprise dans le cours du texte. 
Un tel cas se retrouve avec une transaction établie avec Mermet Fustier. Sur un morceau de papier, déchiré en haut et à droite, on trouve ainsi un encadré qui indique uniquement le nom de l'interlocuteur de Teisseire, suivi d'une liste d'entrées. Il s'agit d'achats effectués par Teisseire, pour lesquels il indique la nature et le montant, avec la somme en fin de note, ainsi que la mention du paiement, daté du samedi 19 janvier. L'année n'apparaît pas. Ce document est clairement un aide-mémoire, permettant au cordier de se rappeler le paiement effectué, éventuellement les fournitures achetées, avec pour repère visuel simple l'encadré autour du nom de cette relation d'affaires. On ne retrouve pas Mermet Fustier au cours du livre de raison : il peut s'agir d'une transaction unique, même si cela paraît étonnant - en effet Jean Teisseire a des cercles professionnels plutôt restreints et suivis. Il se peut également que cette notice soit reliée à des transactions ultérieures, et qu'elle ait été insérée ici par des hasards de conservation que l'on ne peut éclairer totalement.

Fort intéressante aussi est la transaction qui apparaît non pas dans le livre de raison, mais dans un manuscrit de la bibliothèque Ceccano ${ }^{11}$, où l'on trouve un morceau de papier lié de toute évidence à Jean Teisseire et probablement à un livre de raison antérieur (la datation en est la preuve). Cette pièce se présente sous la forme d'un feuillet déchiré à droite, et concerne un compte final établi avec Bertranet Gros. Sont mentionnés le nom de ce dernier ainsi que la somme totale qui reste à payer, 25 florins, et le paiement effectué par Teisseire le $1^{\text {er }}$ octobre 1362. La main de Jean Teisseire n'est pas à mettre en doute, puisqu'au-delà de la graphie similaire on trouve la mention en fin de notice: «en présence de moi, Jean Teisseire ». Cette pièce était très probablement un aide-mémoire, glissé dans un cartulaire a posteriori, qui était antérieur à celui des années 1370

\footnotetext{
${ }^{11}$ Voir supra note 1 pour les références de ces documents.
} 
à 1376. Tout comme la pièce concernant Mermet Fustier, on peut envisager qu'il s'agisse d'un feuillet utilisé alors que le livre n'était pas immédiatement disponible, de manière à y reporter ensuite la notice. Impossible de dire en revanche si cette notice a bel et bien été reportée, en l'absence de ce cartulaire.

Les habitudes scripturales du marchand lui viennent des mondes administratifs, notariés et comptables : il est à la croisée de ces sphères sociales, et son parcours individuel traverse en réalité de nombreuses problématiques partagées par les petites élites urbaines dans leur ensemble. Les techniques qu'il met en place et leurs buts, s'ils ne sont pas nouveaux, n'en sont pas moins d'une grande efficacité pratique et d'une certaine sophistication. Ainsi, il s'inscrit dans les pratiques scripturales des marchands et petites élites urbaines, développées à partir du XII ${ }^{\mathrm{e}}$ et $\mathrm{XIII}^{\mathrm{e}}$ siècle surtout. Ces écritures marchandes traduisent une forme de volonté de gestion par l'écrit, qui passe par une organisation hiérarchique du propos, une mise en liste qui joue un rôle essentiel dans un processus d'administration domestique et de remémoration. Une telle organisation, à la fois pratique et visuelle, et une telle administration entraînent des habitudes en termes de classement, de tri, de sélection et d'archivage que ce dossier illustre ${ }^{12}$. Cette notion de classement, associée à celle de mémoire, est essentielle dans la compréhension de ces écritures, mais aussi

\footnotetext{
${ }^{12}$ Sur les pratiques de classement, il est utile de dépasser la période concernée par Jean Teisseire et de comparer avec les pratiques des périodes modernes et contemporaines. Un article particulièrement éclairant permet de comprendre le rapport entre les particuliers et leurs archives. Voir ainsi Claudine Dardy, «De la paperasserie à l'archive : l'administration domestique », dans Par écrit, ethnologie des écritures quotidiennes, dir. Daniel Fabre, Paris, Éd. de la Maison des sciences de l'homme, 1997, p. 187-200. Sur les pratiques de l'écrit plus généralement, en Provence et ailleurs, les perspectives anthropologiques sont toujours enrichissantes également. Voir ainsi Jack Goody, La Raison graphique : la domestication de la pensée sauvage, Paris, Éditions de Minuit, 1979, ainsi que id., Pouvoirs et savoirs de l'écrit, Paris, La Dispute, 2007.
} 
dans celle de leur arrangement visuel, notamment et surtout dans les ruptures graphiques telles qu'on les a étudiées (listes, délinéarisation du texte), de façon à transformer la pensée en un objet saisissable et par là même, modifiable. C'est ainsi toute la question du rapport entre pensée et écrit qui est posée, rejoignant alors les considérations philosophiques et celles des cognitivistes ${ }^{13}$.

13 Voir ainsi Georg Wilhelm Friedrich Hegel, Encyclopédie des sciences philosophiques. Vol. 3 : Philosophie de l'esprit, Paris, Vrin, 1988, paragraphe 462 : "C'est dans les mots que nous pensons ». Voir également Henri Bergson, La pensée et le mouvant, Paris, PUF, 1975, ou encore Maurice Merleau-Ponty, Signes, Paris, Gallimard, 1960. 